\title{
AOR
}

Selected Papers of \#AolR2020:

The $21^{\text {st }}$ Annual Conference of the

Association of Internet Researchers

Virtual Event / 27-31 October 2020

\section{ALGORITHMIC PRODUCTION BEYOND SILICON VALLEY}

Dan M. Kotliar (organizer)

The Hebrew University of Jerusalem and Stanford University

Rivka Ribak

University of Haifa

Shazeda Ahmed

UC Berkley

Jonathan Roberge

Institut National de la Recherche Scientifique (INRS)

Marius Senneville

Institut National de la Recherche Scientifique (INRS)

The last decade has seen a proliferation of research on algorithms. Algorithms were shown to influence the content we see online (Gillespie 2018), our chances of getting a job or a loan (Pasquale 2013), our relationships with our friends, colleagues or bosses (Bucher 2018), and even how we express and understand ourselves (Turow and Couldry 2018). Algorithms were also shown to affect our identities (Cheney-Lippold 2017), our choices (Yeung 2017), and our autonomy (Rouvroy 2013), and to mirror, and at times exacerbate social inequalities (Noble 2018; Benjamin 2019; Buolamwini and Gebru 2018; Eubanks 2018). At the same time, scholars have begun to examine the ties between algorithms and culture (Seaver 2017; Christin 2018; Ribak 2019; Seyfert and Roberge 2016), describing algorithms as products of complex socioalgorithmic assemblages (Gillespie 2016, 24), with often very local socio-technical histories (Kitchin 2017, 16; Seaver 2017).

However, while the power of algorithms is becoming unmistakable, the spatial trajectories through which algorithms operate, and the specific socio-cultural contexts in which they arise have been largely overlooked. That is, research overwhelmingly focuses on American companies (and particularly, on a handful of Silicon Valley companies) and on the effects their algorithms have on Euro-American users. But

Suggested Citation (APA): Kotliar, D., Ribak, R., Ahmed, S., Roberge, J., Senneville, M. (2020, October). Algorithmic Production Beyond Silicon Valley. Panel Presented at AoIR 2020: The 21 th Annual Conference of the Association of Internet Researchers. Virtual Event: AolR. Retrieved from http://spir.aoir.org. 
algorithms are in fact being developed in various geographical locations, and they are being used in highly diverse socio-cultural contexts. Moreover, companies, engineers, and even algorithms themselves often move from one geographic location to the next. That is, research on algorithms tends to disregard the heterogeneous contexts in which algorithms arise, the spatial aspects of algorithmic production, and the effects various cultural settings have on the production of algorithmic systems.

Focusing on case studies from China, Israel, and Canada, we will ask: How do developers view information privacy at the intersection of local and global flows of ideas? How cultural identities and cross-cultural encounters construct notions of privacy? How is algorithmic bias and discrimination understood and acted upon in China? What symbolical and material resources were invested in making Canada's Al hubs? And how do Israeli tech companies use their algorithms to overlook culture and profile their "Others"? Focusing on algorithmic production across three continents, this panel offers to think beyond the paradigm of Silicon Valley, and to aim towards a more nuanced, culturally sensitive approach to the study algorithms.

Discussant: Angele Christin, Stanford University

\section{REFERENCES}

Benjamin, Ruha. 2019. Race After Technology: Abolitionist Tools for the New Jim Code. New York, NY: John Wiley \& Sons.

Bucher, Taina. 2018. If...Then: Algorithmic Power and Politics. Oxford, UK: Oxford University Press. https://doi.org/10.1093/oso/9780190493028.001.0001.

Buolamwini, Joy, and Timnit Gebru. 2018. "Gender Shades: Intersectional Accuracy Disparities in Commercial Gender Classification." In Proceedings of the 1st Conference on Fairness, Accountability and Transparency, edited by Sorelle A Friedler and Christo Wilson, 81:77-91. Proceedings of Machine Learning Research. New York, NY, USA: PMLR. http://proceedings.mlr.press/v81/buolamwini18a.html.

Cheney-Lippold, John. 2017. We Are Data: Algorithms and The Making of Our Digital Selves. New York: NYU Press.

Christin, Angèle. 2018. "Counting Clicks: Quantification and Variation in Web Journalism in the United States and France." American Journal of Sociology 123 (5): 1382-1415. https://doi.org/10.1086/696137.

Eubanks, Virginia. 2018. Automating Inequality: How High-Tech Tools Profile, Police, and Punish the Poor. New York, NY: St. Martin's Press.

Gillespie, Tarleton. 2016. "Algorithm." In Digital Keywords: A Vocabulary of Information Society and Culture, edited by Benjamin Peters, 18-30. Princeton, N.J.: Princeton University Press. 
- 2018. Custodians of the Internet: Platforms, Content Moderation, and the Hidden Decisions That Shape Social Media. New Haven, CT: Yale University Press.

Kitchin, Rob. 2017. "Thinking Critically about and Researching Algorithms." Information, Communication \& Society 20 (1): 14-29. https://doi.org/10.1080/1369118X.2016.1154087.

Noble, Safiya U. 2018. Algorithms of Oppression: How Search Engines Reinforce Racism. New York: NYU Press.

Pasquale, Frank. 2013. "The Emperor 's New Codes: Reputation and Search Algorithms in the Finance Sector." In Governing Algorithms Conference, 1-86. New York.

Ribak, Rivka. 2019. "Translating Privacy: Developer Cultures in the Global World of Practice." Information, Communication \& Society 22 (6): 838-53. https://doi.org/10.1080/1369118X.2019.1577475.

Rouvroy, Antoinette. 2013. "The End(s) of Critique : Data-Behaviourism vs. DueProcess." In Privacy, Due Process and the Computational Turn: The Philosophy of Law Meets the Philosophy of Technology, edited by M Hildebrandt and K De Vries, 143-69. New York: Routledge.

Seaver, Nick. 2017. "Algorithms as Culture: Some Tactics for the Ethnography of Algorithmic Systems." Big Data \& Society 4 (2). https://doi.org/10.1177/2053951717738104.

Seyfert, Robert, and Jonathan Roberge. 2016. "What Are Algorithmic Cultures?" In Algorithmic Cultures: Essays on Meaning, Performance and New Technologies, edited by Robert Seyfert and Jonathan Roberge, 1-26. New York: Routledge.

Turow, Joseph, and Nick Couldry. 2018. "Media as Data Extraction: Towards a New Map of a Transformed Communications Field." Journal of Communication 68 (2): 415-23. https://doi.org/10.1093/joc/jqx011.

Yeung, Karen. 2017. "Hypernudge': Big Data as a Mode of Regulation by Design." Information, Communication \& Society 20 (1): 118-36. https://doi.org/10.1080/1369118X.2016.1186713. 


\title{
MATERIALIZING PRIVACY IN LOCAL AND GLOBAL DEVELOPER COMMUNITIES
}

\author{
Rivka Ribak \\ University of Haifa
}

Contemporary Al systems execute value-based decisions (Zarsky, 2016) which must be attentive to national and transnational laws and regulations, and sensitive to cultural norms and values (JafariNaimi, 2018). This poses a challenge to the global companies that develop them - specifically, how privacy is to be designed when it is a contested and contextual concept, ridden by cultural variation (Mulligan, Koopman \& Doty, 2016; Nissenbaum, 2004). Developers in these companies are assigned with interpreting global architectures into vernacular interfaces, and local practices into worldwide platforms and infrastructures. As inadvertent cultural producers (Neff, 2012), their daily work involves negotiating tensions between local and global, ethical and technical ideas and values. Yet ideas and values are not easily nor transparently inscribed into cultural products; so rather than copy and paste, the developers translate them (Frenkel, 2005; Latour, 1987). The challenges to privacy that they identify, and the solutions they develop, both mediate and are mediated by the web of local and global interests and practices of which they are a part.

This view of IT workers as socio-technical mediators appears to inform the recent surge in research on developers' values and their perceptions of privacy. Overall, these studies suggest that developers do not prioritize privacy, and are not critical or reflective about the privacy implications of their work. For instance, in interviews with workers in Facebook and Google, Jørgensen (2018) identifies "a disconnect between the internal discourse on freedom of expression and privacy at Google and Facebook, and external concerns relating to these issues" (p. 341); his interviewees believe that privacy and freedom of expression are cherished by their companies, and are to be protected from government threats. While such studies shed light on developers' perceptions and values, we know surprisingly little about these developers and their work environments. The groundbreaking work of Bamberger and Mulligan (2013) constitutes an exception in localizing the production of privacy. Through interviews with corporate privacy leaders in the US, Germany, France and Spain, Bamberger and Mulligan learn about the ways in which companies' Chief Privacy Officers translate legal and regulatory discourse to the company and supervise its implementation in company risk management and product development.

Studies of cross-cultural encounters in high-tech companies shed light on two complementary moves: encounters both accentuate cultural identities and enhance cultural flows. Such tensions appear even more pronounced in imbalanced encounters between workers hailing from developed and developing economies - e.g. a visit of Pakistani software developers to the partnering book publishing company in Denmark (Zahedi \& Babar, 2016), designating the outsourced team as an "offshore." Takhteyev's ethnography of software developers in Rio de Janeiro (2012) highlights flows of ideas and practices between Silicon Valley and "places that can also be aptly described as 'not Silicon Valley'," where the overwhelming majority of developers' work (p. 205). 
Takhteyev characterizes a 'diasporic' situation of peripheral practitioners, who engage simultaneously in the local mainstream culture and the global culture of the practice.

These conceptual threads underlie the following research questions:

How do developers view information privacy at the intersection of local and global flows of ideas and practices?

How are notions of information privacy implicated in cultural identities, and how crosscultural encounters, in turn, construct notions of privacy?

\section{Materializing privacy in developer communities}

In preliminary interviews (Ribak, 2019), I probed at the role of cross-cultural encounters in mediating developers' notions of information privacy. As against the construction of developers as a generic occupational category, the analysis highlighted the role of cultural identity in the ways developers conceive of and commodify user information.

Specifically, the analysis identified four themes that shed light on the intersection of local ideas and practices, cross-cultural encounters, and the production and commodification of users' personal information: (1) the trajectory of the company as it is narrated into a founding myth, and the ways in which changing concepts of privacy are interwoven in its evolution; (2) workers' personal and professional biographies, as narrations of their interests, priorities, and shifting positions within this dynamic matrix; (3) stories about external regulatory forces that impose privacy standards on the one hand and cultural diversity or homogeneity on the other; and (4) observations on how cross-cultural variation and regulation are translated into and intertwined with work practices, rituals and communication formats.

The analysis suggests, then, that developers' ecosystems, as multicultural, global environments, are arenas in which local ideas about privacy are negotiated and take shape. In the proposed presentation I analyze additional interviews with developers from diverse cultural backgrounds, asking how peripheries matter, and how ideas materialize in code.

In a recent piece, titled "how Silicon Valley sets time," Judy Wajcman (2019) draws on interviews with designers, software engineers and product managers to explore how their economic rationality and efficiency express themselves in the scheduling apps they produce. She explains:

"Like all artifacts, electronic calendars [...] are the result of a series of specific decisions made by particular groups of people at particular times and in particular places. As such, technologies are crystallizations of society: they bear the imprint of the people and the social context in which they develop" (2019, p. 1276).

In this spirit, Wajcman perceptively describes her interviewees' hyper-productive culture, and critically observes how their preoccupation with time, as a linear and ownable resource, informs their efforts to quantify and calibrate it and minimize waste. Wajcman makes the point that her interviewees are also users of the technology, although of course - as the very pilgrimage to Palo Alto (an "iconic place" (p. 1286)) 
suggests - these are not ordinary consumers - or even ordinary producers - of calendars. In the presentation I adopt Wajcman's rationale, but attempt to draw production closer to the ground by studying it in "not Silicon Valley" (Takhteyev, 2012). Specifically, I expand the breadth and the depth of the exploratory interviews I conducted in order to compare different "not Silicon Valley" locations in order to make finer distinctions between different peripheral sites.

\section{REFERENCES:}

Bamberger, K. A., \& Mulligan, D. K. (2013). Privacy in Europe. George Washington Law Review, 81(5), 1529-1664.

Frenkel, M. (2005). The politics of translation. Organization, 12(2), 275-301. information society and culture (pp. 18-30). Princeton University Press.

JafariNaimi, N. (2018). Our bodies in the trolley's path, or why self-driving cars must *not* be programmed to kill. Science, Technology, \& Human Values, 43(2), 302-323.

John, N. A. (2011). Representing the Israeli internet. International Journal of Communication, 5, 1545-1566.

Jørgensen, R. F. (2018). Framing human rights. Information, Communication \& Society, 21(3), 340-355.

Latour, B. (1987). Science in action. Harvard University Press.

Mulligan, D. K., Koopman, C., \& Doty, N. (2016). Privacy is an essentially contested concept. Philosophical Transactions of the Royal Society A, 374(2083), 118.

Neff, G. (2012). Venture labor. MIT press.

Nissenbaum, H. (2004). Privacy as contextual integrity. Washington Law Review, 79, 119157.

Ribak, R. (2019). Translating privacy. Information, Communication \& Society, 22(6), 838-853.

Takhteyev, Y. (2012). Coding places. MIT Press.

Wajcman, J. (2019). How Silicon Valley sets time. New Media \& Society, 21(6), 12721289.

Zahedi, M., \& Babar, M. A. (2016). Why does site visit matter in global software development. Information and Software Technology, 80, 36-56.

Zarsky, T. (2016). The trouble with algorithmic decisions. Science, Technology \& Human Values, 41(1), 118-132. 


\section{BEYOND 'BIG DATA SWINDLING': A LITERATURE REVIEW OF CHINESE PERSPECTIVES ON ALGORITHMIC DISCRIMINATION}

Shazeda Ahmed

UC Berkley

As the understanding that algorithms pervade and shape countless aspects of everyday life has gained consensus in academia and mass media, the corollary realization that they can have discriminatory outcomes has likewise become widely accepted-yet the question of how bias is perceived and responded to outside of North American and Western European cultural contexts remains neglected. Algorithms can be discriminatory in other geographies, cultures, and languages, which makes understanding the phenomena that people outside of Western democracies identify as demonstrative of algorithmic bias valuable in advancing this field of study. China presents a rich site for this type of inquiry. International perceptions of China have coalesced around the image of a technological juggernaut pouring funding into developing artificial intelligence (Al) applications, whose government uses much of this technology to surveil its citizens and curb their freedom of expression, and whose technology industry is now exporting these tools. The prevalence of these narratives has to date obscured fundamental questions of how internal debates on what constitutes inappropriate uses of technology in China are playing out- debates that must be accounted for if conversations about China's technological development are to evolve.

Assuming that China's government and technology firms will seek more control in international tech policy-making processes ${ }^{1}$ to match the state's purported status as a global leader in Al development, researchers have an imperative to investigate how issues of algorithmic discrimination are viewed within the country. Such studies can explicate the domestic Chinese discourse around regulation of algorithmic systems, and contextualize how Chinese policymakers might act in international tech policymaking arenas. This paper offers a first step towards building this understanding, in the form of a literature review of Mandarin sources spanning academic, tech industry, news media, and policy texts that address algorithmic bias (算法歧视, suànfă qíshi).

The paper's core source texts comprise Chinese research papers, policy documents, and news articles centered around discussions of terms and concepts in direct translation from English, such as algorithmic discrimination and black box algorithms (黑 箱算法, hēixiāng suànfă). The literature review also surfaced new words and phrases that have emerged in China to describe related issues, e.g., the roughly translated "big data swindling" (大数据杀熟, dà shùjù shā shú) to describe price discrimination against frequent users of digital platforms whose massive data profiling capabilities enable companies to exploit users' differential willingness to pay in China's mostly oligopolistic

\footnotetext{
${ }^{1}$ Gross, Anna et al. 2019. Chinese tech groups shaping UN facial recognition standards. Financial Times. https://www.ft.com/content/c3555a3c-0d3e-11ea-b2d6-9bf4d1957a67.
} 
tech ecosystem. ${ }^{2}$ How does this range of authors define algorithmic discrimination and identify real-world examples of it in China and beyond? What are the proposed solutions to problems of algorithmic discrimination, and who is tasked with the responsibility of executing them? And finally, do conversations about algorithmic discrimination provide an avenue for discussing sensitive sociopolitical issues in China?

Certain arguments, examples, and calls to action recur across these sources. Examples of algorithmic bias tend to draw from the US context, including frequent references to the racially biased COMPAS pretrial risk and sentencing assessment tool, alongside others such as Google's gender-based discriminatory practices in delivering job advertisements through search and racial biases reproduced in facial recognition systems (Ji 2018). More in-depth examples from China include framing price discrimination in e-commerce and ride-hailing apps as a form of algorithmic bias which state media itself recognizes as such (Yang and Luo 2019, Wen 2018), and elements of the Supreme People's Court "smart court" (智能法院, zhinéng făyuàn) project to integrate Al into courtrooms across China (Sun 2019). Still other sources diverge from critiques of ethnic, gender, and consumer discrimination in arguing that China's algorithmic bias problems stem from a lack of data produced in rural, poor areas of the country (Ji 2018). Citing state-produced data on the lower number of mobile phone users in these regions, this argument posits that the lack of data from non-metropolitan areas is reflected in digital under-representation of, and ultimately discrimination against, these populations. Without directly referencing the literature on "data deserts," this line of reasoning closely mirrors some of the same conclusions and proposed solutions to that framing of the problem.

Comparative exercises across the literature are often followed by imperatives to adopt Western-style educational and commercial practices. Multiple sources gesture to top US universities' course offerings on Al ethics and the social impacts of Al to suggest that Chinese students also require this kind of education in addition to their technical training (Liu and Chi 2019). This view pairs with the acknowledgment that there is a dearth of scholarly attention to algorithmic discrimination in China (Yang and Luo 2019). Similarly, the identification of major US tech companies that are developing internal AI ethics roles and teams highlights how Chinese firms could learn from this precedent (Sun 2019), a solution that falls under the more nebulous call for stricter self-regulation (自律, zilü) within companies (Yan 2019). Tech companies notably make little mention of algorithmic discrimination, or do so superficially as in an "annual trends" report from social media and entertainment company Tencent that listed algorithmic discrimination as a trending tech issue of 2019 alongside net neutrality.

\footnotetext{
2 The literal translation of this term would be more akin to the clunky "big data-enabled killing through familiarity," where the latter portion of the phrase, 杀熟 (shā shú), is a colloquialism used to describe taking advantage of someone one is personally close to in order to benefit oneself, e.g., when seeking employment. While this phrase tends to describe interactions with relatives and friends, when it is used in conjunction with big data the "familiarity" comes from the ways in which companies' massive datasets culled from their customers' online activity enables them to understand those individuals' behaviors and exploit or manipulate them into paying a premium for goods and services.
} 
Elsewhere, Chinese legal scholars compare US anti-discrimination law to what they see as its weaker Chinese counterpart in order to make the case that the latter is illequipped to manage algorithmic discrimination and must therefore be reformed. ${ }^{3}$ Calls for revision of existing data protection laws to include language on algorithmic bias remain unmet (Cui 2019). ${ }^{4}$ Both types of policy revision recommendations avert the suggestion of creating entirely separate laws around the use of algorithmic systems more generally, or drafting of sector-specific regulations.

The growing discourse on algorithmic discrimination in China is itself a challenge to the notion that all Chinese citizens uncritically accept the incursion of algorithmic systems into everyday life. It may also provide the seeds of understanding of what Chinese tech companies, policymakers, and other social institutions value in the development of automated systems. Such a foundation could enable scholarly analyses to look beyond the trinity of fairness, accountability, and transparency that have become omnipresent in much writing about algorithmic bias in the West.

\section{REFERENCES}

Cui, Jingzi [崔靖梓]. 2019. The Crisis to Equal Rights Protection Under Algorithmic Discrimination and Its Responses [算法歧视挑战下平等权保护的危机与应对]. Science of Law (Journal of Northwest University of Political Science and Law) Mar 2019 [法律科学( 西北政法大学学报) 2019 年第3 期]: 29-42.

Ji, Jie [季洁]. 2018. Financial Risk Prevention and Control Under Algorithmic Discrimination [算法歧视下的金融风险防控]. Shanghai Finance Oct 2018 [《上海金融》 2018 年第 10 期]: 60-64.

Liu, Pei and Zhongjun Chi [刘培、池忠军]. Ethical Reflections on Algorithmic Discrimination [算法歧视的伦理反思]. Journal of Dialectics of Nature 41(10) [自然辩证法 通讯]: 16-23.

\footnotetext{
${ }^{3}$ This position might surprise experts on US law given the understanding that the burden of proof for bringing discrimination cases in the United States is often regarded as too high for most plaintiffs to meet. Critiques of how this is further complicated when algorithmic systems become involved have emerged in response to the Department of Housing and Urban Development's proposed rule to protect companies who use algorithmic decision-making tools in the housing industry from being sued for discriminator treatment or outcomes these systems might be found to cause. See: Selbst, Andrew. 2019. A New HUD Rule Would Effectively Encourage Discrimination by Algorithm. Slate. https://slate.com/technology/2019/08/hud-disparate-impact-discrimination-algorithm.html. ${ }^{4}$ Although some drafted laws around data protection address algorithmic recommendation systems and the need to respect users' rights to opt out of having their data collected for this purpose, these protections were likely designed as a response to targeted advertising rather providing false or harmful information rather than the set of issues the literature labels as indicative of algorithmic discrimination. Ramzy, Austin. 2016. China Investigates Baidu After Student's Death From Cancer. New York Times. https://www.nytimes.com/2016/05/04/world/asia/china-baidu-investigation-student-cancer.html.
} 
Sun, $\mathrm{Na}$ [孙那]. 2019. The Construction of Legal Ethics for Artificial Intelligence [人工智 能的法律伦理建构]. Jiangxi Social Sciences Feb 2019 [《江西社会科学》 2019 年第2 期 ]: 15-23.

Wen, Jing [温婧]. 2018. Using Big Data to "Swindle" Is Routine for Ecommerce [用大数 据

“杀熟”, 电商的套路都在这了]. Times Weekly [时代周报].

http://baijiahao.baidu.com/s?id=1595270398443964710.

Yan, Jing [严景]. 2019. Algorithmic Bias in Artificial Intelligence and Its ResponsesFrom the Perspective of Gender Discrimination in a Certain Company's Al Resume Screening System

[人工智能中的算法歧视与应对一以某公司人工智能简历耖选系统性别歧视为视角]. Legality Vision (14) [《法制博览》14期]: 127-128.

Yang, Chengyue and Xianjue Luo [杨成越、罗先觉]. 2018. A Preliminary Study into Comprehensive Governance of Algorithmic Discriminations [算法歧视的综合治理初探]. Science and Society (10) [《科学与社会》第 10 期]: 1-12. 


\title{
DATA ORIENTALISM: ON THE ALGORITHMIC CONSTRUCTION OF THE NON-WESTERN OTHER
}

\author{
Dan M. Kotliar \\ The Hebrew University of Jerusalem and Stanford University
}

While the social consequences of algorithms have been systematically discussed (Noble 2018; Benjamin 2019; Eubanks 2018; O’Neal 2016; Bucher 2018; Gillespie 2016), research on algorithms tends to assume that the companies that write and run big data algorithms are American, and accordingly, that the global spread of such algorithms is unidirectional - from "the West" onwards. Similarly, while recent research has shown that algorithms stem from specific socio-cultural contexts (Seaver 2017; Christin 2018; Ribak 2019; Shestakofsky 2017), and that data tends to mirror the social surroundings from which it was extracted (Angwin et al. 2016; Crawford 2016), the distances and differences between the people who develop such algorithms and the users their algorithms affect remain overlooked.

Moreover, while recent research tends to compare algorithmic powers to colonial powers (Couldry and Mejias 2019; Mann and Daly 2019), the move from the colonial gaze (Yegenoglu 1998; Said 1995) to the algorithmic gaze (Graham 2010) has yet to be discussed. This paper aims to fill these gaps and ask: How do companies use their algorithms to "see" and profile their Other? What happens to this Saidian knowledge/power nexus when knowledge about the Other is algorithmically produced? And what is the role of the algorithmic gaze in the expansion of "data colonialism"? This paper will answer these questions by focusing on the case study of an Israeli data analytics company and its attempts to sell its algorithmic products to companies in East Asia.

This paper is based on a 5-year ethnographic study of the Israeli data analytics industry that included 40 semi-structured interviews, participant observations, online content analyses, and more. Particularly, this presentation will focus on the case study of Extractive - an Israeli company that provides user profiling algorithms to companies in Singapore, China, and the Philippines. I will show that Extractive's view of the Other stems from multiple opposing-but-complimentary perspectives: from their "culture agnostic" algorithms that can allegedly overlook culture, race, or locality; from the names of their categories that stem from a globalist, techno-elitist ethos; and finally, from their more traditional, Orientalist attempts to capitalize from the otherness of the Other.

I will accordingly argue that this algorithmic gaze is simultaneously a continuation of the colonial gaze and its exact opposite. It is a gaze that disregards culture, but at the same time highlights cultural differences; a gaze that generates countless hyper-individuated identities but that also categorizes people into a handful of supposedly universal categories. A gaze that potentially offers softer ways of seeing (Cheney-Lippold 2017; Lash and Lury 2007), but that falls back into and is based on more "traditional", racist worldviews. I will argue that these opposing-but-complimentary perspectives work together to create Extractive's expansionary vision, to pave their way into Other 
territories, or at the very least, to help them secure their place on the right side of the "big data divide" (Andrejevic 2014). That is, I will show that through their multi-focal view of the Other, this company is creating its own "imaginary cartography of the internet" (Marchart 1998) as a territory that is divided between data collectors and data subalterns - those who see and those who are being seen - while technologically and discursively placing themselves on the "right" side of that map.

Thus, this paper follows Milan and Treré's call to move past the universalist view of datafication (Milan and Treré 2019), but it also challenges the presumption that algorithmic power flows from its centers in the West out to the global peripheries. In fact, as this paper will demonstrate, the algorithmic gaze is dependent upon different actors across different geographic locations, as well as on the dynamic interactions between them. And so, while recent discussions on algorithms and their power overwhelmingly focus on Euro-American companies, we should keep in mind the multi-directional flow of such powers, as well as the multi-cultural relations that sustain them.

\section{REFERENCES}

Andrejevic, Mark. 2014. "The Big Data Divide." International Journal of Communication 8: 1673-89.

Angwin, Julia, Jeff Larson, Surya Mattu, and Lauren Kirchner. 2016. "Machine Bias." ProPublica. 2016. https://www.propublica.org/article/machine-bias-riskassessments-in-criminal-sentencing.

Benjamin, Ruha. 2019. Race After Technology: Abolitionist Tools for the New Jim Code. New York, NY: John Wiley \& Sons.

Bucher, Taina. 2018. IF...THEN: Algorithmic Power and Politics. Oxford, UK: Oxford University Press.

Cheney-Lippold, John. 2017. We Are Data: Algorithms and The Making of Our Digital Selves. New York: NYU Press.

Christin, Angèle. 2018. "Counting Clicks: Quantification and Variation in Web Journalism in the United States and France." American Journal of Sociology 123 (5): 1382-1415. https://doi.org/10.1086/696137.

Couldry, Nick, and Ulises A. Mejias. 2019. The Costs of Connection: How Data Is Colonizing Human Life and Appropriating It for Capitalism. Stanford, CA: Stanford University Press.

Crawford, Kate. 2016. "Can an Algorithm Be Agonistic? Ten Scenes about Living in Calculated Publics." Science, Technology \& Human Values 41 (1): 77-92. https://doi.org/10.1177/0162243915589635.

Eubanks, Virginia. 2018. Automating Inequality: How High-Tech Tools Profile, Police, and Punish the Poor. New York, NY: St. Martin's Press. 
Gillespie, Tarleton. 2016. "Algorithm." In Digital Keywords: A Vocabulary of Information Society and Culture, edited by Benjamin Peters, 18-30. Princeton, N.J.: Princeton University Press.

Graham, Stephen D. 2010. "Interrupting the Algorithmic Gaze? Urban Warfare and US Military Technology." In Observant States." Geopolitics and Visual Culture, edited by and Klaus J. Dodds. MacDonald, Fraser, Rachel Hughes.

Lash, Scott, and Celia Lury. 2007. Global Culture Industry: The Mediation of Things. Malden and Cambridge: Polity.

Mann, Monique, and Angela Daly. 2019. "(Big) Data and the North-in-South: Australia's Informational Imperialism and Digital Colonialism." Television and New Media 20 (4): 379-95. https://doi.org/10.1177/1527476418806091.

Marchart, Oliver. 1998. "The East, the West and the Rest: Central and Eastern Europe between Techno-Orientalism and the New Electronic Frontier." Convergence 4 (2): 56-75. https://doi.org/10.1177/135485659800400208.

Milan, Stefania, and Emiliano Treré. 2019. "Big Data from the South(s): Beyond Data Universalism." Television and New Media 20 (4): 319-35. https://doi.org/10.1177/1527476419837739.

Noble, Safiya U. 2018. Algorithms of Oppression: How Search Engines Reinforce Racism. New York: NYU Press.

O'Neal, Cathey. 2016. Weapons of Math Destruction: How Big Data Increases Inequality and Threatens Democracy. New York: Broadway Books.

Ribak, Rivka. 2019. "Translating Privacy: Developer Cultures in the Global World of Practice." Information, Communication \& Society 22 (6): 838-53. https://doi.org/10.1080/1369118X.2019.1577475.

Said, Edward W. 1995. Orientalism: Western Conceptions of the Orient. New York: Penguin Books.

Seaver, Nick. 2017. "Algorithms as Culture: Some Tactics for the Ethnography of Algorithmic Systems." Big Data \& Society 4 (2). https://doi.org/10.1177/2053951717738104.

Shestakofsky, Benjamin. 2017. "Working Algorithms: Software Automation and the Future of Work." Work and Occupations 44 (4): 376-423.

Yegenoglu, Meyda. 1998. Colonial Fantasies: Toward a Feminist Reading of Orientalism. Cambridge, UK: Cambridge University Press. 


\section{THE GOSPEL OF "WE THE NORTH" OR HOW TO BUILD AN AI HUB OUTSIDE OF THE U.S.}

Jonathan Roberge

Institut National de la Recherche Scientifique (INRS)

Marius Senneville

Institut National de la Recherche Scientifique (INRS)

When, in 2019, a first Canadian team rose to win an NBA championship (the Toronto Raptors), it was accompanied by this novel slogan and sense of pride: "We the North". By itself, such motto is surprising as it indicates a strong, yet diffuse identity where the city and the country are replaced by a powerful, if hard to describe "way of life". A similar phenomenon can be observed with the emergence of the no-less widely adopted call for a "Sillicon Valley of the North". Indeed, Toronto and Montreal have both seen artificial intelligence (Al) hubs emerged around star researchers like Geoffrey Hinton (UofT) and Yoshua Bengio (UdM) and the subsequent interest of companies like Google, Samsung and Uber to leverage Canada's noted availability of machine learning (ML) specialists (Metz, 2017b). Figuring how and why this decentering of technological development away from California was made possible requires to focus on the relevant entrepreneurial and institutional translators as well as their symbolical and material resources. Because many of these developments and strategies rest on the unchallenged assumption that $\mathrm{Al}$ is a force for the "betterment of humankind", their impacts, including on Canadian culture and society, have yet to be fully investigated (Roberge et al., 2019).

Three elements are central to this particular new political economy of Al. First, it is indispensable to acknowledge the past and current contributions of Canadian institutions. This started with CIFAR's commitment in the 1980s, a time when the technique was deemed unfashionable, and is continued today with the C $\$ 125$-million Pan-Canadian Al Strategy, or the C $\$ 100$-million invested by the province of Quebec, of which $80 \%$ has been committed to Bengio-led Montreal Institute for Learning Algorithms (MILA). Second, the MILA itself is significant in the way its very mandate calls for the intermingling of corporate, academic and government actors (Etzkowitz \& Leidesdorff 2000). This participates in an ecological mentality where, following what Slaughter \& Rhodes have termed "academic capitalism", institutional domains blur and hybridize with one another (2010). Third, it should be noted how Al developments are inseparable from a specific ethos or model of "open science" (Leonelli 2013). Researchers see the sharing of information as progress in and of itself. The obligation to choose to pursue a career in either academia or industry becomes less of an issue once research-inclined corporate labs allow, if not actively encourage the publication of research results in scientific journals and conferences. Knowing the shortage of qualified personnel, such open science practices are instrumentally adopted to navigate the "war to attract talents", often going as far as offering dual affiliation (Metz, 2017a). Unrestricted circulation of people and ideas thus allows for companies to track the best of university research and to reach scientists wherever they are, may that be close to the North Pole as in the cases of Montreal and Toronto. 
Elucidating the rise of $\mathrm{Al}$ in the Canadian context implies not only to look at the political economy involved but also the discursive practices that convoyed them (Roberge et al., forthcoming). When discussed, the risks of Al have been addressed through the selfregulation of technology firms, as was the case for instance with Google's involvement with OpenAl in the US (Grygiel \& Brown, 2019). In Canada, the federal government, Quebec and Ontario each have developped their approaches to ethical Al. Quebec and Montreal have both signed the Montreal Declaration for a Responsible Development of Artificial Intelligence, while Ontario, conversely, has yet to adopt the comparable Toronto Declaration. The Federal Government has adopted impact assessment guidelines on its internal use of Al (McKelvey \& Macdonald, 2019). What is it then that these initiatives have in common that enables Al ethics to occupy such a central stage? For problematizations to develop into symbolical justifications, fears are to be addressed, interpretations are to be tempered, and sensitivities are to find a language by which they can be communicated. As Greene et al. note, "Building a moral background for ethical design is partly about shaping public perception, providing the concepts through which Al/ML can be understood" (2019: 8).

The Montreal Declaration, for instance, is the result of a two-year process of consultation with diverse actors from public and private sectors. Problematically, its guiding principles never question whether Al technologies are safe, if specific forms should in fact be developed, or whether certain surveillance technologies should be made illegal. The fact of the matter is that the Montreal Declaration's value statements and core principles are so broad that they do not even specifically address Al. It calls for instance for a development of Al that "must contribute to the realization of a just and fair society"; or, yet again, that "must eliminate relationships of domination between groups and people based on differences of power, wealth, or knowledge" (IA Responsible, 2018). That the automation of knowledge-production is the sole definition of ML, the reason why people invest time and money, and the way that firms are able to create such metamorphoses within power and capital distribution, are simply ignored.

As a conclusion, the presentation will attempt at understanding what there is up North in Canada as emblematic of broader algorithmic deployment of late: dense, yet diffuse spatial implementation; the construction of material as well as (because of) symbolically-rich environment. In Montreal and Toronto, the mantra is "governance at a distance", i.e. to navigate the complexities of the present, it is deemed better to aim for a horizon that is as remote as possible and hope for the best. The Quebec government has, for example, no data privacy reform planned, nothing to say about facial recognition, and only a vague understanding of the consequences Al will have on the job market and higher education. As it turns out, real investments are mostly about techchauvinism, that is yet another attempt at filling-in what could only remain a sort of "we the north" empty-signifier. 


\section{REFERENCES}

Alwani, K., \& Urban, M. C. (2019). Governing the Future: Creating standards for artificial intelligence and algorithms. The Mowat Centre.

https://munkschool.utoronto.ca/mowatcentre/governing-the-future-creating-standardsfor-artificial-intelligence-and-algorithms/

Cardon, D., Cointet, J.-P., \& Mazières, A. (2018). La revanche des neurones. Réseaux, 211(5), 173-220.

Etzkowitz, H., \& Leydesdorff, L. (2000). The dynamics of innovation: From National Systems and "Mode 2" to a Triple Helix of university-industry-government relations. Research Policy, 29(2), 109-123.

Grygiel, J., \& Brown, N. (2019). Are social media companies motivated to be good corporate citizens?. Telecommunications Policy, 43(5), 445-460.

https://doi.org/10.1016/j.telpol.2018.12.003

Al responsible. (2018). Montreal Declaration for a Responsible Development of Artificial Intelligence. https://www.montrealdeclaration-responsibleai.com/

Kirkwood, I. (2019). Samsung opens second Montreal-based Al lab, moves into Mila. BetaKit. https://betakit.com/samsung-opens-second-montreal-based-ai-lab-moves-intomila/

Leonelli, S. (2013). Why the Current Insistence on Open Access to Scientific Data? Big Data, Knowledge production and the Political Economy of Contemporary Biology. Bulletin of Science and Technology Studies, 33(1-2), 6-11.

McKelvey, F., \& Macdonald, M. (2019). Artificial Intelligence Policy Innovations at the Canadian Federal Government. Canadian Journal of Communication, 44(2).

Metz, C. (2017a). Tech Giants Are Paying Huge Salaries for Scarce Al Talent. Medium. https://medium.com/the-new-york-times/tech-giants-are-paying-huge-salaries-forscarce-ai-talent-ac6b19c92813

Metz, C. (2017b). For Google, the Al talent race leads straight to Canada. Wired. https://www.wired.com/2017/03/google-ai-talent-race-leads-straight-canada/

Roberge, J., Morin, K., \& Senneville, M. (2019). "Deep Learning's Governmentality: The Other Black Box" in The Democratization of Artificial Intelligence, A. Sudmann (eds.), Bielefeld, Transcript Verlag, pp. 123-142.

Roberge, J., Senneville, M., \& Morin, K. (Forthcoming). How to Translate Artificial Intelligence? Myths and Justifications in Public Discourse. Big Data and Society.

Slaughter, S., \& Rhoades, G. (2010). Academic capitalism and the new economy: Markets, state, and higher education. Johns Hopkins Univ. Press. 